\title{
Using Electron Diffraction Techniques, CBED and N-PED to measure Strain with High Precision and High Spatial Resolution
}

\author{
J.L. Rouvière ${ }^{1-2}$, Y. Martin ${ }^{1-2}$, N. Bernier ${ }^{3}$, M. Vigouroux ${ }^{3}$, D. Cooper ${ }^{3}$, J.M. Zuo ${ }^{4}$ \\ 1. Univ. Grenoble Alpes, INAC-SP2M, F-38000 Grenoble, France \\ 2. CEA, INAC-SP2M, F-38000 Grenoble, France \\ 3. CEA, LETI, Minatec campus, 17 rue des Martyrs, 38054 Grenoble Cedex 9, France \\ 4. Univ Illinois, Dept Mat Sci \& Engn, 1304 W Green St, Urbana, IL 61801 USA
}

Changes in the lattice parameters, i.e., introduction of strain, can modify material properties greatly. For instance, the band structure is modified with strain and this leads to changes in the transport or optical properties. In microelectronic devices, strain has been used to improve the mobility of charge carriers since 2003, with strain as low as $0.7 \%$ improving mobility by $50 \%$ [1]. Transmission Electron Microscopy (TEM) is presently the only technique that can measure the strain in individual nano-objects with high spatial resolution (about $1 \mathrm{~nm}$ ) and high precision (about $10^{-4}$ ). Here we focus on recent developments we have made in two electron diffraction techniques: Off-axis Convergent Beam Electron Diffraction (CBED) and Nanobeam Precession Electron Diffraction (N-PED). Off-axis CBED can give 3D maps of the complete 3D strain tensor but it is computationally and experimentally demanding. In contrast, N-PED is a straightforward and precise technique, but it is limited to the projected 2D strain.

In off-axis CBED the originality of our approach is to use both the deficient HOLZ lines of the transmitted beam and the excess HOLZ lines of the diffracted beams to measure the strain [2]. Using Bloch wave calculated CBED patterns as tests, we could retrieve 7 out of the 9 components of the deformation gradient tensor F. In particular, the volume of the cells can be determined (Fig. 1b). By using two different electron beam directions engendering an angle of $22^{\circ}$, we show that it is possible to determine the whole tensor F. In addition, the method can also be extended to the analysis of split HOLZ lines that allow measuring the variations of the strain tensor along the electron beam.

Depending on the convergence angle $(\alpha)$, the diffraction spots look like more dots or disks and the diameter of the incident beam varies (Fig. 1). When $\alpha$ is about $2 \mathrm{mrad}$, the diffracted disks do not have a uniform intensity (Fig. 2f), which can complicate accurate determination of the position of the disks. It is why in Nanobeam Electron diffraction (NBED) $\alpha$ is reduced in order to have spots with more uniform intensities (Fig. 2c) [3]. As these intensity variations are very sensitive to thickness, chemical composition and orientation, it turns out that the positioning of the disk centers is not very robust. This is why we introduced the precession of the beam [4]: the incident beam is tilted by an angle $\alpha_{p}$ and rotated around the original incident direction and a "descan" of the beam is applied after the sample in order to bring back the diffracted spot to their original positions (Fig. 1g-1h). The advantages of introducing precession are manifold: (i) uniformity in the disk intensity, (ii) presence of more diffracted spots, (iii) possibility to work with a relatively large convergence angle in order to reduce the beam diameter (Fig. $1 \mathrm{j}-1 \mathrm{k}$ ); all this leads to an improved precision with a smaller probe [4]. Classically, PED is used in crystallography problems with $\alpha_{\mathrm{p}}$ greater than $1^{\circ}$, in order to have diffracted intensities that approaches kinematical calculations. But in this condition, the probe diameter is increased significantly. As we want to avoid this probe diameter increase $\alpha_{\mathrm{p}}$ below $0.5^{\circ}$ is used in the technique N-PED. Best results were obtained on a FEI TITAN microscope equipped with a probe Cs-corrector and a $2 \mathrm{kx} 2 \mathrm{k}$ CCD camera. Very smooth maps of the four components of the projected deformation tensor can be obtained with NPED (Fig.2). As in all diffraction techniques, the main drawbacks of N-PED for acquiring 2D maps are the slow speed and the large amount of data. A major advantage of diffraction-based techniques is to be 
able to analyze samples of non-uniform thickness and non-uniform composition along the electron beam. This will be illustrated by studying core shell nanowires (NWs) (Fig. 11 and $1 \mathrm{~m}$ ).

[1] T Ghani et al, IEDM Techn. Dig. (2003) p. 11.6.1.

[2] Y Martin et al, accepted in Ultramicroscopy.

[3] A. Béché et al, Ultramicroscopy 131 (2013) 10.

[4] J.L. Rouvière et al, Appl. Phys. Lett. 103 (2013) 241913.
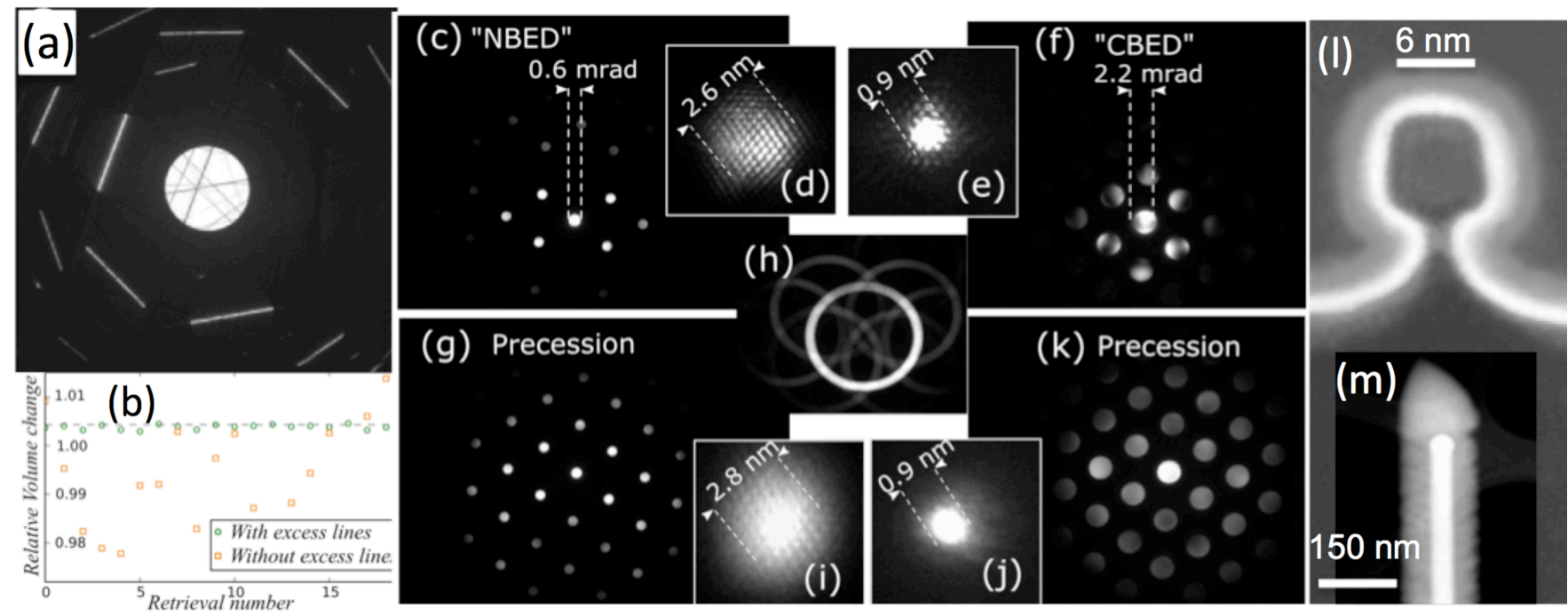

Figure 1. (a) Experimental CBED pattern observed in Si along [651, 441,31]. $\alpha=10$ mrad. Both the transmitted and diffracted beams are used in the fitting of strain. (b) 18 different retrievals of the unit cell volume using a unique CBED pattern that was fitted 18 times with different starting values. The volume of the deformed crystal is retrieved only with the use of the excess lines. (c-g-f-k) Diffraction patterns obtained in different conditions, without ( $\mathrm{c}$ and $\mathrm{f}$ ) or with precession ( $\mathrm{g}$, h and $\mathrm{k}$ ), with a slightly parallel beam (c and g,h) or a more convergent beam (f and k). (d-e-i-j) The associated images of the electron probe when going through Si. (h) Diffraction pattern similar to (g) but with descan off. (l) STEM image of tri-gate device composed of a Si NW surrounded by a TiN gate. (m) Core shell NW, composed of a Ge core surrounded with an amorphous SiN shell. Ge is dilated radially and compressed along its growth axis.
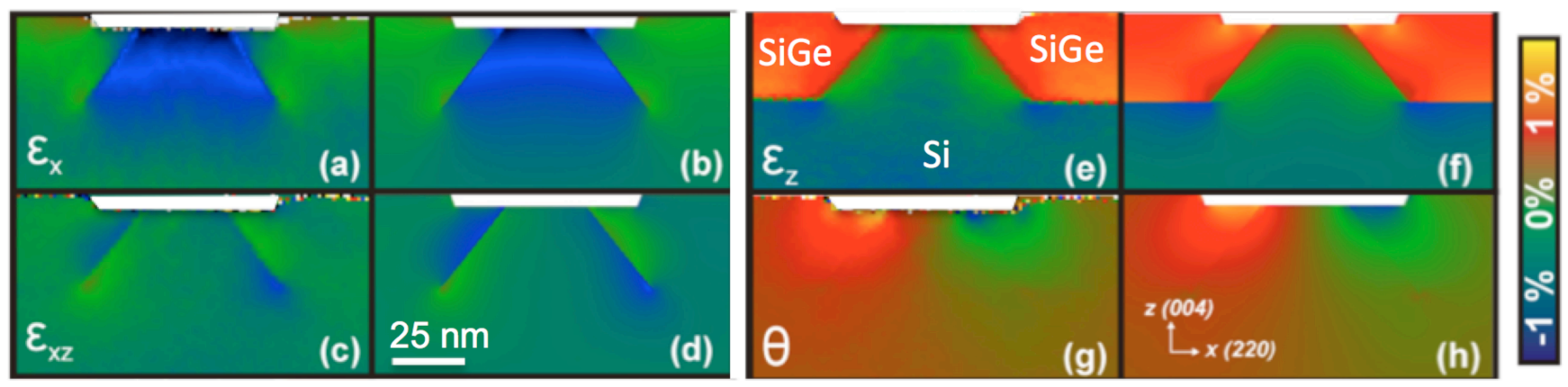

Figure 2. Strain maps obtained in a device containing $\mathrm{SiGe}$ stressors and a $\mathrm{Si}_{3} \mathrm{~N}_{4}$ layer above the $\mathrm{SiO}_{2}$ layer (the whited boxes) above the Si channel. (a-c-e-g) Experimental maps obtained with N-PED. (b-d-f-h) Maps obtained by Finite Element simulations. Note how the strain maps are smooth and are well reproduced by simulations where a $-1.9 \mathrm{GPa}$ stress is introduced in the $\mathrm{Si}_{3} \mathrm{~N}_{4}$ layer. 